\title{
BACKPRESSURE BASED TRAFFIC SIGNAL CONTROL CONSIDERING CAPACITY OF DOWNSTREAM LINKS
}

\author{
Shenxue $\mathrm{HAO}^{1^{*}}$, Licai YANG${ }^{2}$, Yunfeng $\mathrm{SHI}^{3}$, Yajuan $\mathrm{GUO}^{4}$ \\ ${ }^{1}$ School of Transportation and Logistics Engineering, Shandong Jiaotong University, Jinan, China \\ 1,2,3,4School of Control Science and Engineering, Shandong University, Jinan, China
}

Received 27 October 2016; revised 26 February 2017; accepted 5 April 2017

\begin{abstract}
Congestion is a kind of expression of instability of traffic network. Traffic signal control keeping traffic network stable can reduce the congestion of urban traffic. In order to improve the efficiency of urban traffic network, this study proposes a decentralized traffic signal control strategy based on backpressure algorithm used in Wi-Fi mesh networks for packets routing. Backpressure based traffic signal control algorithm can stabilize urban traffic network and achieve maximum throughput. Based on original backpressure algorithm, the variant parameter and penalty function are considered to balance the queue differential and capacity of downstream links in urban traffic network. For each traffic phase of intersections, phase weight is computed using queue differential and capacity of downstream links, which fixed the deficiency of infinite queue capacity in original backpressure algorithm. It is proved that the extended backpressure traffic signal control algorithm can maintain stability of urban traffic network, and also can prevent queue spillback, so as to improve performance of whole traffic network. Simulations are carried out in Vissim using Vissim COM programming interface and Visual Studio development tools. Evaluation results illuminate that it can get better performance than the backpressure algorithm just based on queue length differential in average queue length and delay of traffic network.
\end{abstract}

Keywords: traffic control, queuing network, stability, traffic signal control, backpressure algorithm, penalty function.

\section{Notations}

EBP - extended backpressure algorithm;

MBP - modified backpressure algorithm;

$a, b, c, d$,

$e, f, g, h$ - denote the road segments linked with intersection, which can store vehicles;

$A_{a}(t)$ - the exogenous arriving vehicles of node $a$ from other nodes;

$B$ - a positive constants;

$c_{b}$ - capacity of node $b$;

$c_{r}$ - remaining capacity;

$c_{r, b}$ - remaining capacity of node $b$;

$d_{a b}(t)$ - the number of vehicles departing from node $a$ to $b$;

$f_{a b}$ - traffic flow from node $a$ to node $b$ responding to a traffic phase $p_{j}$;

$F_{i}$ - traffic flow set;

$h$ - road segments $h$ linked with intersection;

$h(a, b)$ - a binary function, the function result is 1 or -1 according to values of $a$ and $b$; $h^{*}$ - target value of penalty function;

$J$ - junction set;

$J_{i}-$ the $i$ th junction of traffic network;

$L(-)$ - Lyapunov function;

$N_{i}$ - node set of junction $J_{i}$;

$N_{i}^{\prime}$ - node set of neighbouring junction $J_{i}$;

$N_{i}^{i n}$ - in-node set, a subset of $N_{i}$ consisting of upstream nodes of all traffic flows in a junction;

$N_{i}^{\text {out }}$ - out-node set, a subset of $N_{i}$ consisting of downstream nodes of all traffic flows in a junction;

$P_{i}$ - traffic phase set of junction $J_{i}$;

$p_{j}$ - the $j$ th phase of $P_{i}$;

$p^{*}$ - the selected phase to be activated in next time slot;

$p_{1}, p_{2}$,

$p_{3}, p_{4},-$ traffic phases of intersection;

$q_{a}$ - number of vehicles on node $a$ waiting for passing through junction;

${ }^{\star}$ Corresponding author. E-mail: haoz2000@163.com 
$q_{b}$ - number of vehicles on node $b$ waiting for passing through junction;

$q_{a b}$ - number of vehicles on node $a$ waiting for moving to node $b$;

$q_{b c}$ - number of vehicles on node $b$ waiting for moving to node $c$;

$\Delta q_{a b}$ - sum of queue length differential between vehicle flows $f_{a b}$ and $f_{b c}$;

$Q^{u}$ - the queues set on upstream node;

$Q^{d}$ - queue set of downstream node;

$t$ - time slot;

$T_{a b}(t)$ - turn ratio of $A_{a}(t)$ driving into $q_{a b}$ at time $t$;

$\mathbf{U}(t)$ - queue vector at time $t$;

$U_{i}(t)$ - the $i$ th compnent of queue vector $\mathbf{U}(t)$;

$V$ - control parameter variable;

$V_{a b}$ - product of remaining capacity and weight coefficient;

$w_{a b}$ - weight of traffic flow $f_{a b}$;

$w_{p}(t)$ - weighted pressure differential of traffic phase $p$;

$\mathbb{E}\{-\}$ - expectation of a random variable;

$\alpha_{a b}$ - coefficient of node $a$ to $b$ computing pressure of node $a$;

$\varepsilon$ - a constant;

$\gamma_{a}-$ the weighted coefficient of node $a$ to compute $V$;

$\mu_{a b}\left(p_{j}\right)$ - the number of vehicles transferred from node $a$ to $b$ when phase $p_{j}$ is activated;

$\mu_{a b}(p)$ - the number of vehicles transferred from node $a$ to $b$ when phase $p$ is activated;

$\Pi_{a}$ - pressure of node $a$;

$\Pi_{b}$ - pressure of node $b$.

\section{Introduction}

Congestion is one of major problems of urban traffic system. Traffic control strategy plays an important role to improve traffic efficiency and balance traffic load. In 1960s, fixed-time traffic control methods are proposed based on off-line historical data, such as TRANSYT (Robertson 1969) and MAXBAND (Little et al. 1981), different optimal timing plans are selected at different time of a day. This kind of strategy cannot deal with fluctuation of traffic demand, since the traffic parameters are predefined. Then, actuated control methods utilize real-time traffic information from detectors on roads to adjust green time for responding real-time traffic demand. However, to actuated control, there is a considerable influence on delay time in detection of sparse traffic conditions (Araghi et al. 2015), and it is applied mainly at isolated intersections (Cesme, Furth 2014). Adaptive traffic control strategies are proposed to adjust signal timing plans based on online traffic information for responding to real-time traffic demand, such as SCOOT (Hunt et al. 1981) and SCATS (Lowrie 1982). These methods can response traffic fluctuation using detector inputs, historical trends and predictive models (Abdelghaffar et al. 2016). Although adaptive traffic control systems have been implied on real urban traffic networks, the centralized controllers obstruct its further development. Urban traffic control system is a classical complex giant system, involving many intersections, roads and vehicles. Centralized traffic control policies are usually based on global information of traffic network, cannot achieve ideal control effect due to complicated calculations and dynamic traffic conditions. Centralized signal timing schemes are computed according to current traffic conditions, but be implied in next cycle, when traffic situation may have changed already (Cesme, Furth 2014).

For fixing these deficiencies, there is a consensus of researchers that distributed traffic control policy is ideal alternative. In distributed traffic control system, signalized intersections are viewed as smart agents, and each agent determines traffic phase switching action according to current local information. Distributed traffic control methods can achieve better performance and adaptation for real-time dynamic traffic conditions (Wu et al. 2014; McKenney, White 2013).

As a completely distributed traffic control scheme, backpressure algorithm based traffic signal control strategies are considered by more researchers, which has been mainly applied in communication networks and introduced into traffic control system recently. Traffic signal control algorithm based on backpressure computes pressure of each traffic phase, and chooses a phase with maximum pressure to activate. Each intersection determines active phase independently according to local traffic information in distributed manner. The original backpressure algorithm introduced in traffic control system can lead to maximum network throughput and guarantee stability of traffic network (Wongpiromsarn et al. 2012).

However, backpressure based traffic signal control algorithm has strong assumptions that all queued vehicles' routing rates are known, and each queue has infinite capacity. These assumptions are hardly to be met in practice (Varaiya 2013). For example, when queue of downstream link is nearly full and the responding phase is activated, queue spillback may occur, which may cause congestion to propagate in traffic network. The existing backpressure based traffic signal control algorithms compute phase pressure to determine active phase according to queue length and routing rates. Traffic conditions of downstream links are neglected. In this paper, we propose an extended backpressure based traffic signal control algorithm considering control parameter $V$ and penalty function, which is inspired by Neely $(2006,2010)$ researching on energy optimal control for wireless networks. The EBP considers trade-off of pressure differential and traffic status of downstream links to prevent queue spillback and improve performance of whole traffic network. The main contributions of our EBP can be summarized as below. Firstly, the EBP is a distributed traffic control strategy. Each intersection of traffic network determines active traffic phase independently according to local traffic information. Secondly, the EBP just requires queue length 
of upstream and downstream links of each phase, and capacity of downstream links, instead of routing rates of all queued vehicles. Thirdly, the control parameter $V$ and penalty function can trade off queue differential and congestion status of downstream links, avoiding spillback on downstream links.

\section{Related works}

Backpressure based routing algorithm is first proposed by Tassiulas and Ephremides (1992). Backpressure algorithm is usually used in wireless multihop networks as an optimal strategy for resource allocation. It has many features including throughput optimality, achievable adaptive resource allocation and simplicity (Jiao et al. 2016). Wongpiromsarn et al. (2012) first adopts backpressure algorithm to solve traffic control problems, and develop a traffic signal control strategy in completely distributed manner. It is proved that backpressure algorithm in traffic network can ensure global optimality as leading maximum network throughput.

Since backpressure based traffic signal control algorithm can maximize throughput of traffic network in completely distributed manner, many researchers pay more attention to this algorithm and get lots of fruits. These papers are focus on three issues, such as pressure computation method, influence from vehicle routing rate, and signal control approach. Varaiya (2013) presents a maximum pressure traffic control strategy based on backpressure algorithm. At each intersection, active phase is selected depending on local queue length, mean turn ratios and saturation rates. Considering the influence from routing rate of queued vehicle on links, Gregoire et al. (2014) present a backpressure traffic signal control algorithm with unknown certain routing rates and estimated aggregated queue length, where vehicle routing information can be detected using detectors on dedicated lanes. In their latter research work, a routing model of traffic network is established with partial controllable vehicles to be used for pressure computation (Gregoire et al. 2016). With communication technology development, more information can be obtained from vehicular network. A multicommodity backpressure algorithm for traffic light control is proposed, where all vehicles' routes are known (Zaidi et al. 2016). In order to improve efficiency of traffic network, route guidance technology is integrated with traffic signal control based on backpressure algorithm (Taale et al. 2015). Le et al. propose cyclic phase backpressure control policy with on-line estimation of turning fraction and measurement of queue size (Le et al. 2015). However, the problem of unrealistic strong assumption that all links have infinite capacity is still unsolved. For infinite queue capacity issue, Gregoire et al. (2015) propose a normalized pressure computation method considering fairness at low traffic density. Xiao et al. (2014) also consider link capacity on computing phase pressure in their paper. Although link capacities are considered for computing phase pres- sure, traffic conditions of downstream links are not taken into count, that is, remaining capacity of downstream link should be considered for preventing queue spillback on downstream links.

These research works mentioned above emphasize pressure computation method based on queue length or queue length differential with different routing rate information. In addition, two kinds of backpressure based signal control approaches are also considered, such as fixed cycle time backpressure algorithm and time slotted backpressure algorithm (Taale et al. 2015). In this paper, we adopt the time slotted traffic control approach, since fixed cycle time control is hardly to meet traffic demand or fluctuation in time. Further, the pressure computation method need consider more realistic factors affecting traffic phase switching. Therefore, we propose a time slotted backpressure based traffic signal control algorithm, considering remaining capacity of downstream links. A control parameter $V$ and penalty function are introduced into backpressure based traffic control algorithm, which are usually used in wireless network. In wireless network, based on original backpressure algorithm Neely introduce control parameter $V$ and penalty function to trade off the minimum power and average end-to-end network delay (Neely 2006). To get better performance, a variable $V$ algorithm is proposed to achieve optimal routing decision in $\mathrm{Wi}-\mathrm{Fi}$ mesh network with queue backlog under control (NúñezMartínez et al. 2011, 2012). Inspired by the parameter $V$ and penalty function used in Wi-Fi mesh network, in this paper we utilize control parameter variable $V$ to trade off queue length differential (pressure differential) and congestion status of downstream links for traffic phase choosing, considering remaining capacity of downstream links in pressure computation. Then, the optimal phase selection is not only according to queue length of each link, but also considering current traffic condition of intersection, such as the congestion status of downstream links.

\section{Traffic network modelling}

In this section, we model road network as a queuing network. Intersections are viewed as junctions that can switch the right of way for each vehicle movement. Road segments between two adjacent intersections are considered as nodes storing vehicles from upstream junction. Nodes in queuing network can be classified into three disjoint groups, i.e. ingress nodes, exit nodes and internal nodes. For the sake of simplicity, we assume that vehicles can only enter traffic network through ingress nodes and depart from exit nodes. That is to say, internal nodes have no other vehicles arrival except vehicles from upstream node. Shown as Figure 1, nodes $\left\{N_{1}, N_{3}, N_{7}, N_{3}^{\prime}, N_{5}^{\prime}, N_{7}^{\prime}\right\}$ are ingress nodes, $\left\{N_{2}, N_{4}, N_{8}, N_{2}^{\prime}, N_{4}^{\prime}, N_{8}^{\prime}\right\}$ are exit nodes, $\left\{N_{5}, N_{6}\right\}$ equals to $\left\{N_{1}^{\prime}, N_{2}^{\prime}\right\}$, are internal nodes. Ingress nodes have no capacity constraint, that is, infinite capacity. Exit nodes have no queued vehicles, on which arrived vehicles leave network immediately. 


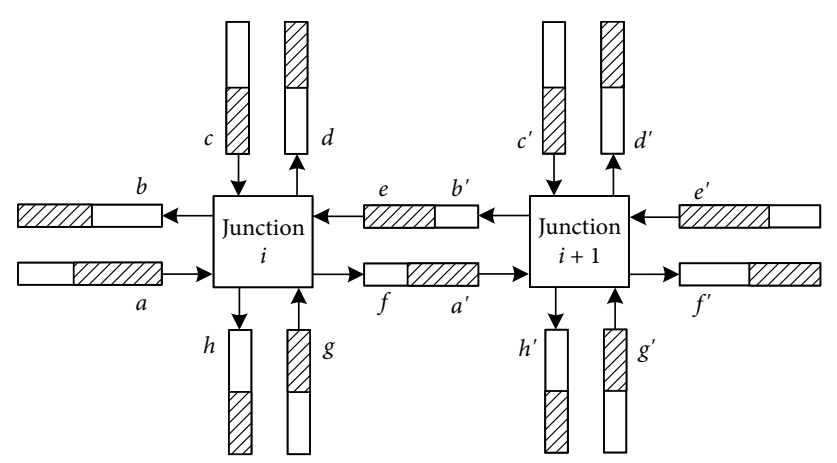

Figure 1. Two adjacent junctions

Let $J$ denote junction set, each $J_{i}$ includes three sets $N_{i}$, $F_{i}$ and $P_{i}$, representing node set, flow set and traffic phase set of $J_{i}$, where a vehicle flow $f_{a b}$ means that vehicles on node $a$ can pass through junction to node $b . N_{i}$ is classified into two subsets: out-node $N^{\text {out }}$ and in-node $N^{\text {in }}$. A flow $f_{a b}$ satisfies condition that $a \in N_{i}^{i n}$ and $b \in N_{i}^{\text {out }}$. There may have several vehicle flows on one node, i.e. left-turn, right-turn and straight vehicle flow. Traffic phase is defined as a group of vehicle flows that have the right of way to pass through junction simultaneously, that is $p_{j} \in P_{i}$ and $f_{a b} \in p_{j}, a \in N_{i}^{i n}, b \in N_{i}^{\text {out }}$. We assume that each traffic phase has dedicated lanes. Note that left-turnlane and straight-lane is corresponding to the left-turnphase and straight-phase respectively; right-turn-lane is not controlled by traffic signal in this paper.

In queuing network control, time is slotted. We assume that the traffic signal phase switches at beginning of slotted time $t$, during each time slot, vehicles entering a node will drive into dedicated lanes immediately. Let $A_{a}(t)$ denote the exogenous arrivals from other nodes, $d_{a b}(t)$ represent the number of vehicles moving from node $a$ to $b$, $T_{a b}(t)$ is turn ratio of exogenous arrivals, that is, there are $T_{a b}(t) \cdot A_{a}(t)$ vehicles added in $q_{a b}(t)$ during time slot $t$. Since right-turn-lane is not controlled by traffic signal, the right-turn-lane does not generate a queue. For the sake of simplicity, we neglect the influence from right-turn vehicles in this paper. Then, queue length of each flow on node $a$ can be computed as follows:

$$
q_{a b}(t+1)=q_{a b}(t)+T_{a b} \cdot A_{a}(t)-d_{a b}(t) .
$$

For example, there are three vehicle flows and three lanes on each link shown as Figure 2, i.e. left-turn-flow (lane 1), straight-flow (lane 2) and right-turn-flow (lane 3). Intersection has four traffic phases: $p_{1}=\{(a, f),(e, b)\}$, $p_{2}=\{(a, d),(e, h)\}, p_{3}=\{(g, d),(c, h)\}, p_{4}=\{(c, f),(g, b)\}$, that is to say, when phase $p_{1}$ is activated vehicles on node $a$ can pass through junction to node $f$ and vehicles on node $e$ can pass through junction to node $b$, vehicles on lane 3 of node $a$ and $e$ can drive into node $h$ and $d$ freely. The others traffic phases are in the similar fashion.

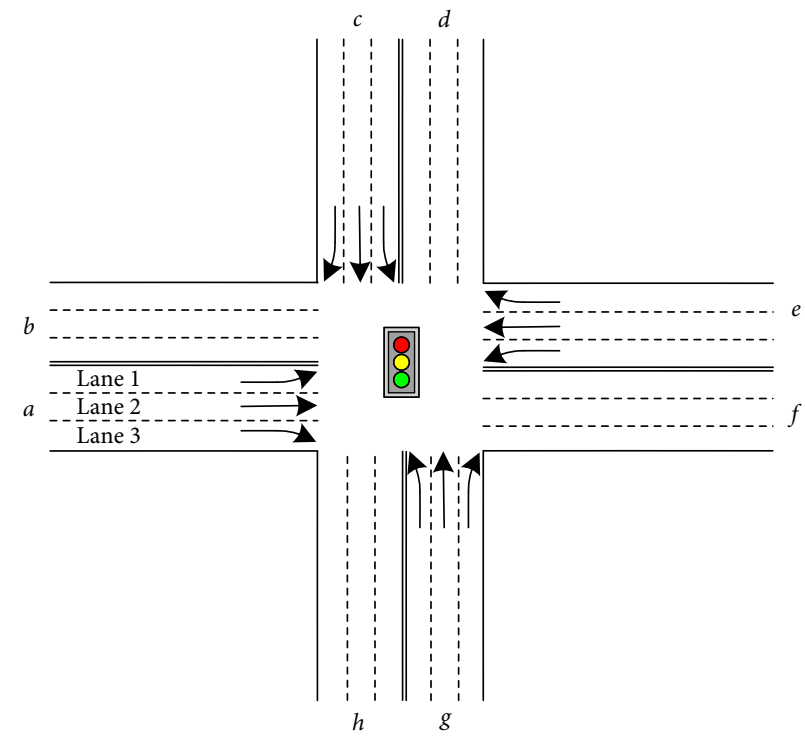

Figure 2. Intersection with four traffic phases

\section{EBP in traffic control}

\subsection{Original backpressure algorithm}

The idea of backpressure algorithm in traffic control is that each junction chooses a traffic phase with maximum weighted pressure differential to activate for achieving maximum throughput of traffic network. The steps of original backpressure algorithm are described as follows:

1) For each junction, compute the pressure of each node with a linear pressure function as follows.

$$
\Pi_{a}(t)=\alpha_{a b} \cdot q_{a}(t)
$$

where: $\alpha_{a b}$ is coefficient $\left(\alpha_{a b}=1\right.$ in research by Wongpiromsarn et al. (2012)); $q_{a}(t)$ is the total number of vehicles waiting on node $a$ at beginning of slot $t$.

2) Compute the weight of each vehicle flow by:

$w_{a b}(t)=\max \left(\Pi_{a}(t)-\Pi_{b}(t), 0\right)$.

3) Choose a phase to activate with maximum weighted pressure differential by:

$$
p^{*}=\underset{p_{j} \in J_{i}}{\arg \max } \sum_{(a, b) \in p_{j}} w_{a b}(t) \cdot \mu_{a b}\left(p_{j}\right),
$$

where: $p^{*}$ is the phase to activate in next time slot; $\mu_{a b}\left(p_{j}\right)$ represents the maximum number of vehicles transferred from node $a$ to $b$ when phase $p_{j}$ is activated. The original backpressure algorithm has been proved that can achieve maximum throughout under infinite queue capacity.

However, the present research works do not consider the influence from capacity of downstream links, especially the case that the downstream links have no enough capacity to afford upstream arriving vehicles, although 


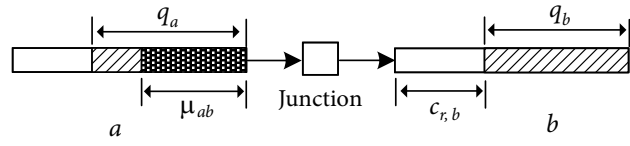

Figure 3. The case of downstream link is nearly full

the very traffic phase has a maximum weighted pressure differential, shown as Figure 3. There is $q_{a}>q_{b}, f_{a b} \in p$ and $\mu_{a b}>c_{r}$. If traffic phase $p$ is to be activated, since the number of transferred vehicles is larger than the remaining capacity of downstream link, spillback may occur. In order to avoid this case and trade off the throughput and congestion, we propose an EBP to improve the performance of traffic network in next section.

\subsection{EBP}

\subsubsection{Pressure differential of traffic flow}

In traffic network, one of vehicle flows waiting for passing through junction on node $a$ will generate pressure to downstream node $b$. There are two queues on node $b$, such as queue of left-turn vehicle flow and straight vehicle flow. We use the sum of queue length differential denote the pressure differential of traffic phase. Let $q_{a b}$ denote the queue length on node $a$ waiting for moving to node $b ; q_{b c}$ denote queue length of one vehicle flow on downstream node $b$, which is moving to node $c$. When the responding traffic phase $p$ is active, vehicles in $q_{a b}$ get the right of way to move from node $a$ to $b$. Therefore, each vehicle flow pressure differential between upstream queue and downstream queue for each traffic phase is computed by the follows equation:

$$
w_{a b}(t)=\sum_{c}\left(q_{a b}(t)-q_{b c}(t)\right),
$$

where: $w_{a b}$ is the weighted pressure differential of vehicle flow $f_{a b}$; the weighted pressure differential of traffic phase $p$ is sum of all vehicle flows weighted pressure differential, computed by equation:

$$
w_{p}(t)=\sum_{(a, b) \in p} w_{a b}(t) \cdot \mu_{a b}(t) .
$$

Based on Equations (6) and (4), we can get the maximum weighted traffic phase to minimum pressure differential of queuing network. However, the modified pressure computation method also cannot avoid queue spillback of downstream node. In next section, we introduce a penalty function to trade off queue differential and congestion to avoid queue overflow.

\subsubsection{Penalty function}

It is well known that backpressure algorithm can guarantee throughput optimality in capacity region. Neely (2006) EBP in his paper, the optimization problem is not only to keep queuing network stability, but also optimize other network parameters or objective function. In this paper, we modify the weight computation method of backpressure algorithm by introducing parameter $V$ and penalty function for preventing spillback of downstream queue. The modified weight computation is as follows:

$$
w_{a b}(t)=\Delta q_{a b}(t)-V_{a b} \cdot h(a, b),
$$

where: the first component $\Delta q_{a b}(t)=\sum_{c}\left(q_{a b}(t)-q_{b c}(t)\right)$, is sum of queue length differential between vehicle flow $f_{a b}$ (vehicles on node $a$ moving to $b$ ) and all $f_{b c}$ (vehicles on node $b$ moving to $c$ ); the second component is penalty function, in this paper, exploits the remaining capacity of downstream link for preventing queues spillback on node $b ; V_{a b}>0$ is a control parameter to trade off minimum queue length differential and remaining capacity; $h(a, b) \in\{1,-1\}$ is a binary function.

As discussed case in Section 3.1, the goal of penalty function is to avoid the queue spillback on node $b$. When $\Delta q_{a b}>0$, traffic phase $p\left(f_{a b} \in p\right)$ is possible to be activated. However, if $\mu_{a b}>c_{r, b}$ that is, downstream node $b$ has no enough capacity to afford the transferring vehicles from $a$, queue of node $b$ will overflow. Therefore, let $h(a, b)=1$ in this case, then $-V_{a b} \cdot h(a, b)<0$, value of $w_{a b}$ decrease. On the other hand, if $\mu_{a b}<c_{r, b}$, that is, downstream link can afford the transferring vehicles from upstream link, we set $h(a, b)=-1$ to increase the value of $w_{a b}$, for preventing from the case of $w_{a b}<0$, even if $\Delta q_{a b}(t)<0$.

When each $\Delta q_{a b}$ of a traffic phase in a junction is more than zero, we take $V_{a b} \cdot h(a, b)$ into count to trade off queue length differential and remaining capacity of downstream link to improve performance of traffic network. Then, value of parameter $V$ is related to the remaining capacity of downstream links and queue length differential. Therefore, we define parameter $V$ of $f_{a b}$ as $V_{a b}=\gamma_{a} \cdot c_{r, b}$, where $c_{r, b}$ is the remaining capacity of downstream node $b$ and $\gamma_{a}$ is the weighted coefficient determined by all queue length of junction.

In summary, in order to prevent queue spillback of downstream node, we modified the computation method of the weight of traffic phase in this EBP as follows:

$$
\begin{aligned}
& w_{p}(t)=\sum_{(a, b) \in p}\left(\sum_{c}\left(q_{a b}(t)-q_{b c}(t)\right)-\right. \\
& \left.V_{a b} \cdot h(a, b)\right) \cdot \mu_{a b}(p) .
\end{aligned}
$$

Shown as Algorithm 1 (Table 1), the proposed algorithm selects phase $p^{*}$ with maximum pressure differential considering remaining capacity of downstream link at junction $J_{i}$ during slot $t$, where parameter $\gamma_{a}$ is the reciprocal of maximum remaining capacity of junction $J_{i}$ in this paper.

The introduced parameter $V$ and penalty function can avoid queue spillback when pressure differential is positive, and also can choose an optimal traffic phase to activate when the pressure differential is negative. In the next section, we prove that the EBP can still achieve stability of traffic network. 
Table 1. Explication of the Algorithm 1

\begin{tabular}{l} 
Algorithm 1: Selection of phase $p^{*}$ with max pressure at junc- \\
tion $J_{i}$ during slot $t$. \\
\hline Input: Information of vehicle queued at each approach of \\
junction $J_{i}$, such as $q_{a b}, q_{b c}$, where $a \in N_{i}^{\text {in }}, b \in N_{i}^{\text {out }}, c \in N_{i+1}^{\text {out }} ;$ \\
$\mu_{a b}(p) ; c_{b}$. \\
Output: $p^{*} \in P_{i}$ is the phase to be activated during slot $t$. \\
\hline$p^{*}=0$; \\
for each phase $p$ in $P_{i}$ \\
$\quad w_{p}(t)=-\infty ;$ \\
for each flow $f_{a b}$ in $p$ \\
$\qquad \quad \Delta q_{a b}(t)=\sum_{c}\left(q_{a b}(t)-q_{b c}(t)\right) ;$ \\
$\qquad c_{r, b}=c_{b}-q_{b} ;$ \\
$\quad V_{a b}=\gamma_{a} \cdot c_{r, b} ;$ \\
$\quad$ if $\mu_{a b}>c_{r, b}$, then $h(a, b)=1 ;$ \\
$\quad$ else $h(a, b)=-1 ;$ \\
$\quad w_{a b}(t)=\Delta q_{a b}(t)-V_{a b} \cdot h(a, b) ;$ \\
end for \\
$w_{p}(t)=\sum_{(a, b) \in p_{j}} w_{a b}(t) \cdot \mu_{a b}\left(p_{j}\right) ;$ \\
end for \\
$p^{*}=$ arg $\max \left(w_{p}(t)\right)$. \\
$p \in P_{i}$
\end{tabular}

\section{Stability analysis of EBP}

In this section, we prove that the proposed algorithm in this paper can achieve network stability. Wongpiromsarn et al. (2012) has proved the stability of original backpressure traffic control algorithm in their paper based on Lemma 1.

Lemma 1. Suppose $\mathbb{E}\left\{U_{i}(t)\right\}<\infty$ for all $i \in\{1,2, \ldots, N\}$ and there exist constants $B>0$ and $\varepsilon>0$ satisfies:

$$
\begin{aligned}
& \mathbb{E}\{L(\mathbf{U}(t+1)-L(\mathbf{U}(t)) \mid \mathbf{U}(t)\} \leq B- \\
& \varepsilon \cdot \sum_{i=1}^{N} U_{i}(t), \forall t \in \mathbb{N}^{0},
\end{aligned}
$$

then the network is table, where queue vector $\mathbf{U}=\left[U_{1}, U_{2}\right.$, ..., $\left.U_{N}\right], L(\mathbf{U}(t))$ is constructed Lyapunov function.

For the sake of simplicity, we assume that $\mathbb{E}\left\{A_{a}(t)\right\}=\lambda_{a}$, where $\lambda_{a}$ is interior to the capacity region of network. Define Lyapunov function $L(\mathbf{U}(t))=\sum_{a, b} q_{a b}(t)^{2}$. Injecting Equation (1) into Lyapunov function, we obtain:

$$
\begin{aligned}
& L(\mathbf{U}(t+1))-L(\mathbf{U}(t))= \\
& \sum_{a, b}\left(q_{a b}(t+1)^{2}-q_{a b}(t)^{2}\right)= \\
& \sum_{a, b}\left(q_{a b}(t)^{2}+\left(T_{a b} \cdot A_{a}(t)-d_{a b}(t)\right)^{2}+\right.
\end{aligned}
$$

$$
\begin{aligned}
& \left.2 \cdot q_{a b}(t) \cdot\left(T_{a b} \cdot A_{a}(t)-d_{a b}(t)\right)-q_{a b}(t)^{2}\right)= \\
& \sum_{a, b}\left(\left(T_{a b} \cdot A_{a}(t)-d_{a b}(t)\right)^{2}+\right. \\
& \left.2 \cdot q_{a b}(t) \cdot\left(T_{a b} \cdot A_{a}(t)-d_{a b}(t)\right)\right) \leq \\
& \sum_{a, b}\left(T_{a b} \cdot A_{a}(t)^{2}+d_{a b}(t)^{2}-2 \cdot T_{a b} \cdot A_{a}(t) \cdot d_{a b}(t)+\right. \\
& \left.2 \cdot q_{a b}(t) \cdot T_{a b} \cdot A_{a}(t)-d_{a b}(t)\right) \leq \\
& B(t)-2 \cdot \sum_{a, b} q_{a b}(t) \cdot\left(d_{a b}(t)-T_{a b} \cdot A_{a}(t)\right),
\end{aligned}
$$$$
\text { where: } B(t)=\sum_{a, b}\left(T_{a b} \cdot A_{a}(t)^{2}+d_{a b}(t)^{2}\right) \text {. }
$$$$
\text { Let } B=\sum_{a, b}\left(T_{a b} \cdot\left(A_{a}^{\max }\right)^{2}+\sup _{p_{j} \in P}\left(d_{a b}(p(t))\right)^{2}\right) \text {, }
$$

where $A_{a}^{\max }$ is the maximum exogenous arrivals, then we get $B(t) \leq B$.

Taking expectation for $L(\mathbf{U}(t+1))-L(\mathbf{U}(t))$, we get:

$$
\begin{aligned}
& \mathbb{E}\{L(\mathbf{U}(t+1))-L(\mathbf{U}(t)) \mid \mathbf{U}(t)\} \leq \\
& B-2 \cdot \sum_{a, b} q_{a b}(t) \cdot \mathbb{E}\left\{d_{a b}(t)-T_{a b} \cdot A_{a}(t)\right\} .
\end{aligned}
$$

Let $\mu_{a}^{\text {in }}(p(t))$ represent the number of vehicles entering node $a, \mu_{a b}^{\text {out }}(p(t))$ represent the number of vehicles departing from $f_{a b}$ when phase $p(t)$ is active. Then, Equation (10) becomes:

$$
\begin{aligned}
& \mathbb{E}\{L(\mathbf{U}(t+1))-L(\mathbf{U}(t)) \mid \mathbf{U}(t)\} \leq \\
& B-2 \cdot \sum_{a, b} q_{a b}(t) \times \\
& \mathbb{E}\left\{\mu_{a b}^{\text {out }}(p(t))-t_{a b} \cdot \mu_{a}^{\text {in }}(p(t)) \mid q(t)\right\}= \\
& B-2 \cdot \sum_{a, b} q_{a b}(t) \times \\
& \mathbb{E}\left\{\mu_{a b}^{\text {out }}(p(t))-\mu_{a b}^{\text {in }}(p(t)) \mid q(t)\right\} .
\end{aligned}
$$

Since $q_{a b}$ denote the queue of upstream node $a, q_{b c}$ denote one of queues in the downstream node $b$, and $q_{a b}$ generate pressure to all the queue of downstream node $b$. For convenient description, let $Q^{u}$ denote the queues set on upstream node, $Q^{d}$ denote the queue set of downstream node. Therefore, Equation (11) becomes:

$$
\begin{aligned}
& \mathbb{E}\{L(\mathbf{U}(t+1))-L(\mathbf{U}(t)) \mid \mathbf{U}(t)\} \leq \\
& B-2 \cdot \sum_{a, b} q_{a b}(t) \times \\
& \mathbb{E}\left\{\mu_{a b}^{\text {out }}(p(t))-\mu_{a b}^{i n}(p(t)) \mid q(t)\right\}= \\
& B-2 \cdot \sum_{\substack{q_{a b} \in Q^{u} \\
q_{b c} \in Q^{d}}}\left(q_{a b}(t)-q_{b c}(t)\right) \times
\end{aligned}
$$




$$
\begin{aligned}
& \mathbb{E}\left\{\mu_{a b}(p(t)) \mid q(t)\right\}= \\
& B-2 \cdot \sum_{a, b}\left(q_{a b}(t)-\sum_{c} q_{b c}(t)\right) \times \\
& \mathbb{E}\left\{\mu_{a b}(p(t)) \mid q(t)\right\} .
\end{aligned}
$$

Inject Equation (5) into Equation (12), we get:

$$
\begin{aligned}
& \mathbb{E}\{L(\mathbf{U}(t+1))-L(\mathbf{U}(t)) \mid \mathbf{U}(t)\} \leq \\
& B-2 \cdot \sum_{a, b}\left(q_{a b}(t)-\sum_{c} q_{b c}(t)\right) \times \\
& \mathbb{E}\left\{\mu_{a b}(p(t)) \mid q(t)\right\}= \\
& B-2 \cdot \sum_{a, b} w_{a b}(t) \cdot \mathbb{E}\left\{\mu_{a b}(v) \mid q(t)\right\} .
\end{aligned}
$$

Therefore, based on Equations (12) and (13) we can conclude that the queuing network is stable under backpressure algorithm with modified pressure computation method. This algorithm also can minimum queue length differential of queuing network, because it chooses the traffic phase with maximum $w_{a b}(t)$, while $\mathbb{E}\{L(\mathbf{U}(t+1))-L(\mathbf{U}(t)) \mid \mathbf{U}(t)\}$ is minimum. For the EBP, Neely (2006) has proved the Lemma 2 as below.

Lemma 2. Lyapunov drift performance optimization. If there are positive constants $V, B, \varepsilon$ such that for all time slots $t$ and all vectors $U(t)$, the one-step conditional Lyapunov drift satisfied:

$$
\begin{aligned}
& \Delta(U(t))+V \cdot E\{h(a, b) \mid U(t)\} \leq B- \\
& \varepsilon \cdot \sum_{a, b} U(t)+V \cdot h^{*},
\end{aligned}
$$

then the system is stable, where $\Delta(U(t)) \triangleq \mathbb{E}\{L(\mathbf{U}(t+1))$ $L(\mathbf{U}(t)) \mid \mathbf{U}(t)\}, h^{*}$ is the target value of penalty function $h(a, b)$. It is clear that Equation (14) is hold when $V$ is bounded. According to the definition of parameter $V_{a b}$ in Section 3.2.2, $V$ is bounded. Therefore, the EBP can also stabilize traffic network.

\section{Simulation}

In this section, we utilize Vissim to study the performance of backpressure algorithms in traffic network. Firstly, we construct a simulation traffic network with 15 intersections and 76 links as shown in Figure 4a, including 16 ingress links and 16 exit links. Vehicles input of ingress links is $1500 \mathrm{veh} / \mathrm{h}$. Each intersection has 4 traffic phases, 4 approaches and 8 links. There are 3 lanes in each link, including left-turn-lane, right-turn-lane and straight-lane, shown as Figure $4 \mathrm{~b}$. There sets static route decision at 10 $\mathrm{m}$ from the start point of each link, this is, vehicles entered a link will determine their route soon, and drive into dedicated lanes based on route decision. Route decisions on one link are shown as Figure 4c. All the roads in traffic network are bi-directional. In order to test performance of backpressure algorithm considering remaining capacity of downstream links, the link length are varied between 120 and $254 \mathrm{~m}$ except for ingress links and exit links. Parameters are obtained from Vissim COM programming interface such as the number of queued vehicle on dedicated lane, vehicle type, etc. Backpressure based traffic signal control algorithms are implemented using Visual Basic 6.0, and control parameters are fed back into Vissim at every time slot.

a)

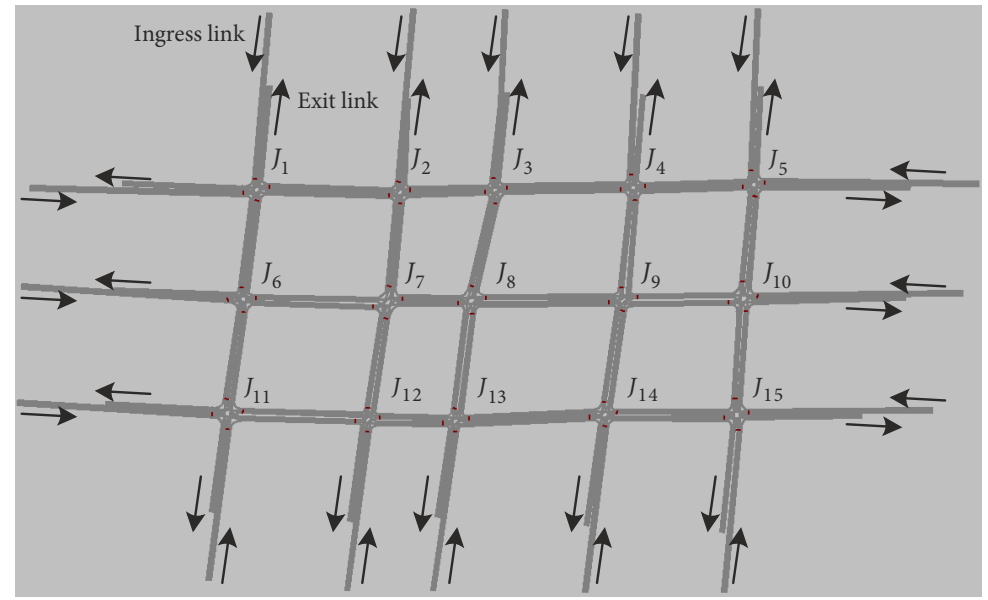

b)

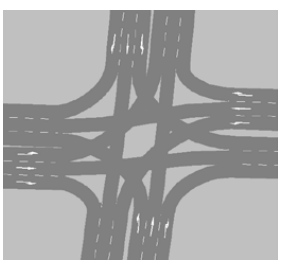

c)

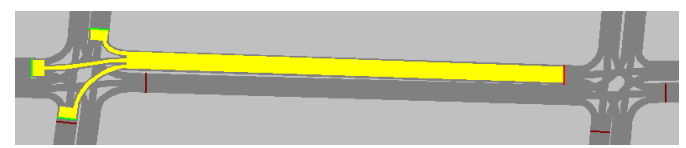

Figure 4. Simulation traffic network in Vissim: a - topology of simulation traffic network; $\mathrm{b}$ - link connection of an intersection; $\mathrm{c}$ - route decision on a link 


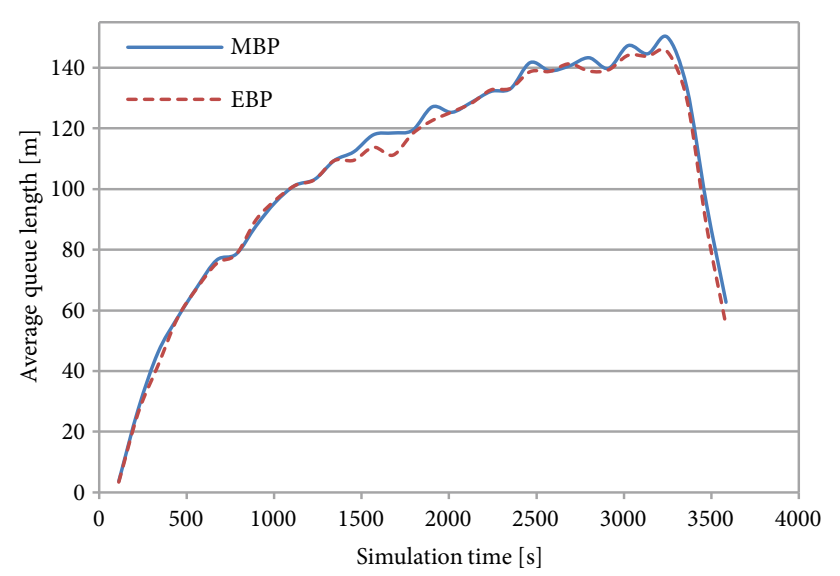

Figure 5. Average queue length of traffic network under different control algorithm

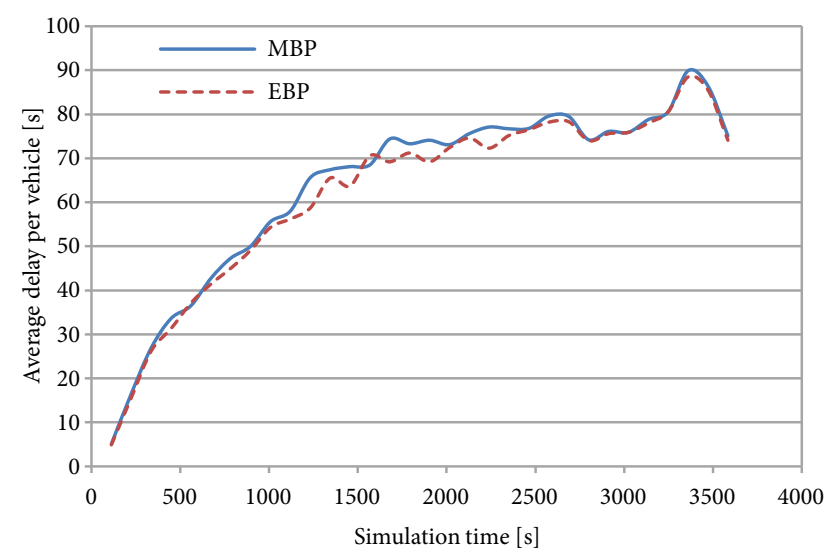

Figure 6. Delay of traffic network under MBP and EBP

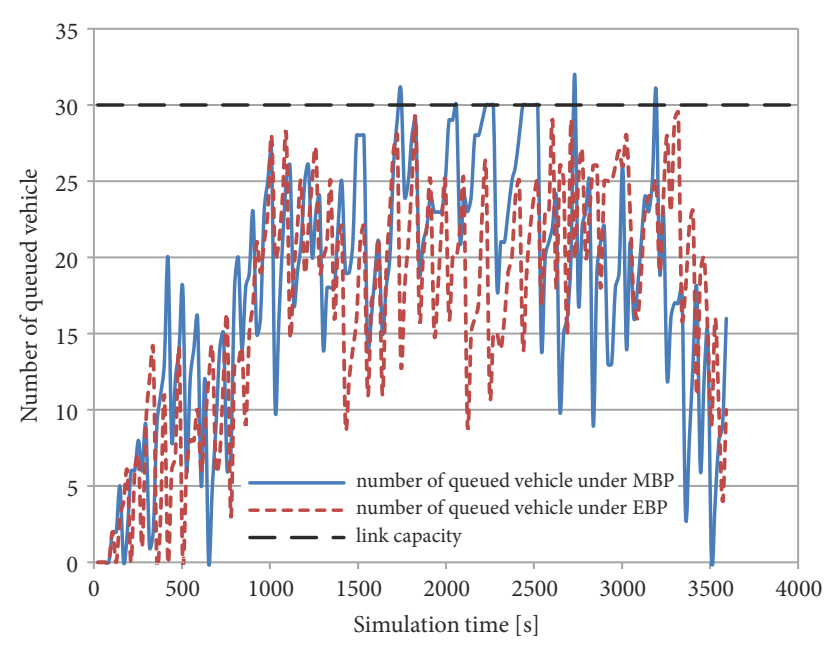

Figure 7. Number of queued vehicle on a link under $\mathrm{EBP}$ and $\mathrm{MBP}$
Two backpressure based traffic signal control algorithms are implemented, one is the MBP in research by Gregoire et al. (2014), and the other is EBP proposed in this paper. For the sake of simplify description, the two backpressure algorithms are named as MBP and EBP respectively.

Results shown in Figure 5 are average queue lengths of the two backpressure algorithms. It is clear that the average queue lengths are same in beginning of simulation, and increase as the number of vehicles in traffic network increasing. Figure 5 illuminates that EBP can get lower average queue length than MBP when number of vehicles in traffic network is large. Figure 6 shows that EBP can get less delay in the same situation.

From Table 2 of the performance of whole traffic network during simulation, EBP can get better performance. Backpressure based traffic control method is a distributed policy for urban road networks. It can determine the switching traffic phase based on the local traffic information especially queue length of each link of intersections. EBP can get better performance, because EBP considers not only the queue length, but also the capacity of downstream links for preventing spillback.

Shown as Figure 7, a link selected from the simulation traffic network, the number of queued vehicles under MBP exceeds link capacity sometimes, spillback may occur in practice, which may result in seriously congestion. Sine EBP considers the capacity of downstream links as multiply of penalty function and variant parameter $V$, it can prevent spillback in traffic network to improve performance of whole traffic network.

\section{Conclusions}

In this paper, we modified computation of traffic phase pressure, and introduced parameter $V$ and penalty function into traffic control based on backpressure algorithm. The proposed decentralized backpressure based traffic control algorithm can stabilize traffic network, and prevent spillback by considering capacity of downstream links. Parameter $V$ and penalty function are introduced into maximum weighted pressure traffic phase choosing, which can balance queue differential and capacity of downstream links, so as to improve performance of whole traffic networks. In this paper, we proved that the proposed EBP can stabilize traffic network, simulation illuminates that the extended backpressure can get better performance.

However, in practice, a traffic network cannot be viewed as a queuing network simply, there are many fac-

Table 2. Performance indexes of traffic network during simulation in Vissim

\begin{tabular}{|l|c|c|c|c|c|c|c|}
\cline { 2 - 8 } \multicolumn{1}{c|}{} & $\begin{array}{c}\text { Average number } \\
\text { of stops }\end{array}$ & $\begin{array}{c}\text { Average speed } \\
{[\mathrm{km} / \mathrm{h}]}\end{array}$ & $\begin{array}{c}\text { Average stopped } \\
\text { delay }[\mathrm{s} / \mathrm{veh}]\end{array}$ & $\begin{array}{c}\text { Average delay } \\
\text { time }[\mathrm{s} / \mathrm{veh}]\end{array}$ & $\begin{array}{c}\text { Total delay } \\
\text { time }[\mathrm{h}]\end{array}$ & $\begin{array}{c}\text { Total stopped } \\
\text { delay }[\mathrm{h}]\end{array}$ & $\begin{array}{c}\text { Total travel } \\
\text { time }[\mathrm{h}]\end{array}$ \\
\hline EBP & 20.034 & 16.201 & 150.624 & 229.841 & 1368.958 & 897.131 & 1979.994 \\
\hline MBP & 21.137 & 15.953 & 154.449 & 236.209 & 1406.887 & 919.917 & 2020.866 \\
\hline
\end{tabular}


tors influencing the computation of traffic pressure, such as vehicle type, number of passengers, urgency degree of different vehicles, transit priority, parking activities, pedestrian, bus stations, even traffic accident. These factors should be considered in practice. For different type of vehicles, there are different vehicle lengths. It results that same number of queued vehicles has different queue length and generate different pressure. Therefore, pressure computation should consider the vehicle types with different length. For considering different vehicle length, the queue length that is used to compute pressure in this paper can be modified as product of the number of vehicles and vehicle length. For considering the number of passengers, researchers investigate traffic signal control algorithms to optimize person delay in traffic network. Based on the EBP, number of passengers in queued vehicles can be introduced into pressure computation to solve the optimal person delay issues. In the similar way, transits in queue also can be weighted to compute a new weighted traffic pressure to solve the transit priority issues. However, the question of how to determine weight of vehicle in queue need to be studied in future works, since the $V$ parameter and penalty function are based on capacity of downstream links, and these factors are independently to link capacity. Some transfer functions between parameter $V$ and the weight of these factors need to be designed. Therefore, investigation for applications of backpressure based traffic signal control algorithm is the main objective of our future works.

In the other hand, the number of queued vehicles on one intersection is considered for pressure computation in this paper. In order to further improve performance of traffic network, coordination of neighbouring intersections should be considered in the future work, since the approaching vehicles from upstream links will generate pressure to the downstream intersections. The variant time slot should also be considered for different traffic conditions in the future work.

\section{Funding}

This work is supported by:

- Scientific Research Foundation of Shandong Jiaotong University, China [Grant No Z201927, Z201928],

- Doctoral Research Initiation Foundation of Shandong Jiaotong University, China [Grant No BS201902022];

- Jinan Self-Developed Innovation Team Project of "20 Policies for Colleges and Universities" Program, China [Grant No 2019GXRC022].

\section{Contribution}

The main contributions of our EBP can be summarized as below.

Firstly, the EBP is a distributed traffic control strategy. Each intersection of traffic network determines active traffic phase independently according to local traffic information.
Secondly, the EBP just requires queue length of upstream and downstream links of each phase, and capacity of downstream links, instead of routing rates of all queued vehicles.

Thirdly, the control parameter $V$ and penalty function can trade off queue differential and congestion status of downstream links, avoiding spillback on downstream links.

\section{Disclosure statement}

There have no any competing financial, professional, or personal interests from other parties.

\section{References}

Abdelghaffar, H. M.; Yang, H.; Rakha, H. A. 2016. Isolated traffic signal control using a game theoretic framework, in 2016 IEEE 19th International Conference on Intelligent Transportation Systems (ITSC), 1-4 November 2016, Rio de Janeiro, Brazil, 1496-1501. https://doi.org/10.1109/ITSC.2016.7795755

Araghi, S.; Khosravi, A.; Creighton, D. 2015. A review on computational intelligence methods for controlling traffic signal timing, Expert Systems with Applications 42(3): 1538-1550. https://doi.org/10.1016/j.eswa.2014.09.003

Cesme, B.; Furth, P. G. 2014. Self-organizing traffic signals using secondary extension and dynamic coordination, Transportation Research Part C: Emerging Technologies 48: 1-15. https://doi.org/10.1016/j.trc.2014.08.006

Gregoire, J.; Frazzoli, E.; De La Fortelle, A.; Wongpiromsarn, T. 2014. Back-pressure traffic signal control with unknown routing rates, IFAC Proceedings Volumes 47(3): 11332-11337. https://doi.org/10.3182/20140824-6-ZA-1003.01585

Gregoire, J.; Qian, X.; Frazzoli, E.; De La Fortelle, A.; Wongpiromsarn, T. 2015. Capacity-aware backpressure traffic signal control, IEEE Transactions on Control of Network Systems 2(2): 164-173. https://doi.org/10.1109/TCNS.2014.2378871

Gregoire, J.; Samaranayake, S.; Frazzoli, E. 2016. Back-pressure traffic signal control with partial routing control, in 2016 IEEE 55th Conference on Decision and Control (CDC), 12-14 December 2016, Las Vegas, NV, US, 6753-6758. https://doi.org/10.1109/CDC.2016.7799309

Hunt, P. B.; Robertson, D. I.; Bretherton, R. D.; Winton, R. I. 1981. SCOOT - a Traffic Responsive Method of Coordinating Signals. Transport and Road Research Laboratory (TRRL) Report LR1014. Crowthorne, Berkshire, UK. 41 p. Available from Internet: https://trl.co.uk/reports/LR1014

Jiao, Z.; Zhang, B.; Li, C.; Mouftah, H. T. 2016. Backpressurebased routing and scheduling protocols for wireless multihop networks: a survey, IEEE Wireless Communications 23(1): 102-110. https://doi.org/10.1109/MWC.2016.7422412

Le, T.; Kovács, P.; Walton, N.; Vu, H. L.; Andrew, L. L. H.; Hoogendoorn, S. S. P. 2015. Decentralized signal control for urban road networks, Transportation Research Part C: Emerging Technologies 58: 431-450. https://doi.org/10.1016/j.trc.2014.11.009

Little, J. D. C.; Kelson, M. D.; Gartner, N. H. 1981. MAXBAND: a program for setting signals on arteries and triangular networks, Transportation Research Record 795: 40-46.

Lowrie, P. R. 1982. The Sydney coordinated adaptive traffic system - principles, methodology, algorithms, in International Conference on Road Traffic Signalling, 30 March - 1 April 1982, London, UK, 67-70. 
McKenney, D.; White, T. 2013. Distributed and adaptive traffic signal control within a realistic traffic simulation, Engineering Applications of Artificial Intelligence 26(1): 574-583.

https://doi.org/10.1016/j.engappai.2012.04.008

Neely, M. J. 2006. Energy optimal control for time-varying wireless networks, IEEE Transactions on Information Theory 52(7): 2915-2934. https://doi.org/10.1109/TIT.2006.876219

Neely, M. J. 2010. Stochastic network optimization with application to communication and queueing systems, Synthesis Lectures on Communication Networks 3(1): 1-211. https://doi.org/10.2200/S00271ED1V01Y201006CNT007

Núñez-Martínez, J.; Mangues-Bafalluy, J. 2012. Distributed Lyapunov drift-plus-penalty routing for WiFi mesh networks with adaptive penalty weight, in 2012 IEEE International Symposium on a World of Wireless, Mobile and Multimedia Networks (WoWMoM), 25-28 June 2012, San Francisco, CA, US, 1-6. https://doi.org/10.1109/WoWMoM.2012.6263779

Núñez-Martínez, J.; Mangues-Bafalluy, J.; Portoles-Comeras, M. 2011. Studying practical any-to-any backpressure routing in Wi-Fi mesh networks from a Lyapunov optimization perspective, in 2011 IEEE Eighth International Conference on Mobile Ad-Hoc and Sensor Systems, 17-22 October 2011, Valencia, Spain, 771-776. https://doi.org/10.1109/MASS.2011.86

Robertson, D. I. 1969. TRANSYT: a Traffic Network Study Tool. Transport and Road Research Laboratory (TRRL) Report LR253. Crowthorne, Berkshire, UK. 37 p. Available from Internet: https://trl.co.uk/reports/LR253

Taale, H.; Van Kampen, J.; Hoogendoorn, S. 2015. Integrated signal control and route guidance based on back-pressure principles, Transportation Research Procedia 10: 226-235. https://doi.org/10.1016/j.trpro.2015.09.072

Tassiulas, L.; Ephremides, A. 1992. Stability properties of constrained queueing systems and scheduling policies for maximum throughput in multihop radio networks, IEEE Transactions on Automatic Control 37(12): 1936-1948.

https://doi.org/10.1109/9.182479

Varaiya, P. 2013. Max pressure control of a network of signalized intersections, Transportation Research Part C: Emerging Technologies 36: 177-195. https://doi.org/10.1016/j.trc.2013.08.014

Wongpiromsarn, T.; Uthaicharoenpong, T.; Wang, Y.; Frazzoli, E.; Wang, D. 2012. Distributed traffic signal control for maximum network throughput, in 2012 15th International IEEE Conference on Intelligent Transportation Systems, 16-19 September 2012, Anchorage, AK, US, 588-595. https://doi.org/10.1109/ITSC.2012.6338817

Wu, Q.; Li, B.; Chen, K. 2014. A multi-agent traffic control model based on distributed system, Sensors \& Transducers 173(6): 60-67.

Xiao, N.; Frazzoli, E.; Li, Y.; Wang, Y.; Wang, D. 2014. Pressure releasing policy in traffic signal control with finite queue capacities, in 53rd IEEE Conference on Decision and Control, 15-17 December 2014, Los Angeles, CA, US, 6492-6497. https://doi.org/10.1109/CDC.2014.7040407

Zaidi, A. A.; Kulcsár, B.; Wymeersch, H. 2016. Back-pressure traffic signal control with fixed and adaptive routing for urban vehicular networks, IEEE Transactions on Intelligent Transportation Systems 17(8): 2134-2143.

https://doi.org/10.1109/TITS.2016.2521424 